\title{
Daño pulmonar asociado al uso de cigarrillos electrónicos-vapeadores
}

\author{
SERGIO BELLO S.*
}

\section{Pulmonary damage associated with the use of electronic cigarettes or vapers}

There has been a lot of discussion about the harmful health effects caused by electronic cigarettes or vapers and their usefulness as a smoking cessation aid. More and more publications appear with deleterious effects on health. This discussion has been straightened in recent years, due to the significant increase in the use of vapers worldwide, especially among adolescents and young adults. In September 2019, the US Center for Disease Control and Prevention warned of a significant number of cases of lung disease associated with the use of electronic cigarettes (EVALI: e-cigarette or vaping associated lung injury). Epidemiologically it was considered an outbreak that as of January 17, 2020 presented 2668 hospitalized patients, with 57 deaths. During the week of September 15, 2019 the peak of hospitalizations for EVALI occurred. The majority were young men. $82 \%$ of them used products with Tetrahydrocanabinoids (THC) and 14\% products with nicotine. In the bronchoalveolar lavage of 51 cases of EVALI, the presence of Vitamin E acetate was found, a product used as a thickener for the elaboration of vaping products containing THC, which makes it a possible causal factor, but it cannot be ruled out the contribution of other toxic compounds. The world's leading scientific societies and World Health Organization have warned of the long-term risks of using electronic cigarettes and recommend their control and regulation.

Key words: Smokers; Smoking cessation; Electronic nicotine delivery system; vaping; Tetrahydrocanabinoids; Lung injury; Vitamin E acetate.

\section{Resumen}

Ha habido mucha discusión sobre los efectos dañinos para la salud producidos por los cigarrillos electrónicos o vapeadores y su utilidad como ayuda para dejar de fumar. Cada vez aparecen más publicaciones con efectos deletéreos sobre la salud. Esta discusión se ha acentuado en los últimos años, por el importante aumento del uso de los vapeadores en todo el mundo, especialmente entre los adolescentes y adultos jóvenes. En septiembre de 2019 el Centro de Control y Prevención de Enfermedades $(C D C)$ de los EE. UU. alertó sobre un importante número de casos de enfermedad pulmonar asociada al uso de cigarrillo electrónico (EVALI: e-cigarette or vaping associated lung injury). Epidemiológicamente se consideró un brote que al 17 de enero, 2020 ha presentado 2.668 pacientes hospitalizados, con 57 fallecidos. Durante la semana del 15 de septiembre 2019 ocurrió el 'peak' de hospitalizaciones por EVALI. La mayoría eran varones jóvenes. El $82 \%$ usó productos con Tetrahidrocanabinoides (THC) y el 14\% productos con nicotina. En el lavado bronquio-alveolar de 51 casos de EVALI se encontró la presencia de acetato de Vitamina E, producto utilizado como espesante para la elaboración de productos de 'vapeo' que contienen THC, lo que lo hace un posible factor causal, pero no se puede descartar el papel de otros compuestos tóxicos. Las principales sociedades científicas del mundo y la OMS han advertido de los riesgos a largo plazo del uso de los cigarrillos electrónicos y recomiendan su control y regulación.

Palabras clave: Fumadores; dejar de fumar; Sistemas electrónicos de administración de nicotina; vapeadores; Tetrahidrocanabinoides; Daño pulmonar; Acetato de Vitamina E.

* Comisión de Tabaquismo. Sociedad Chilena de Enfermedades Respiratorias. 


\section{Introducción}

Los cigarrillos electrónicos son dispositivos que contienen una batería que calienta un líquido, transformándolo en "vapor" y que la persona inhala. A pesar que el "vapor" es en realidad un aerosol, de aquí surge el neologismo de "vapear" y la denominación de "vapeadores" a los dispositivos que permiten "vapear".

Los cigarrillos electrónicos utilizan una bobina metálica como resistencia para calentar y aerolizar una mezcla de glicerina vegetal, propilenglicol, nicotina y agentes saborizantes. El líquido del cigarrillo electrónico es conducido desde un estanque a la bobina por una mecha hecha de algodón, sílice o cerámica, y el usuario activa una corriente eléctrica a través de la bobina presionando un botón o generando un flujo de aire a través del dispositivo ${ }^{1}$. Desde su entrada al mercado hace 15 años, los fabricantes de cigarrillos electrónicos han introducido un sin número de cambios en su diseño que permiten al usuario controlar la composición del líquido, la concentración de nicotina y como se produce la aerolización del líquido, existiendo en la actualidad cigarrillos electrónicos de primera, segunda, tercera y cuarta generación. En forma genérica se han denominado 'Sistemas Electrónicos de Administración de Nicotina' (SEAN) o 'Dispositivos Electrónicos de Liberación de Nicotina' (DELN) $\mathrm{y}$ en la literatura anglosajona se conocen como Electronic Nicotine Delivery System (ENDS).

La seguridad del uso de los SEAN y su utilidad como elementos de ayuda para dejar de fumar, han estado en discusión desde su introducción en los mercados mundiales. Ha habido defensores de su uso, basados en que serían menos dañinos que los cigarrillos convencionales y tendrían alguna utilidad en los fumadores que quieren dejar de fumar. Es así como el Servicio de Salud Pública de Inglaterra lo ha promovido en sus programas de apoyo a la cesación tabáquica $^{2}$. Pero la mayoría de las organizaciones de salud del mundo, partiendo por la Organización Mundial de la Salud ${ }^{3}$ y sociedades científicas de prestigio internacional como la European Respiratory Society (ERS) ${ }^{4}$, Unión Internacional contra la Tuberculosis y Enfermedades Pulmonares (The Union $^{5}$, Foro Internacional de Sociedades Respiratorias (FIT) ${ }^{6}$, Asociación Latinoamericana de Tórax (ALAT) ${ }^{7}$ no lo recomiendan y han solicitado a los gobiernos y tomadores de decisión que se regule su uso. En el mundo ya hay 26 países que han prohibido su comercialización, entre los que destacan Brasil, Uruguay, Panamá, México, Qatar, Arabia Saudita, Tailandia. Recientemente se prohibió en India y en el estado de California de los EE.UU.

Ha habido muchas publicaciones sobre los componentes tóxicos de los cigarrillos electrónicos ${ }^{8,9}$, sus efectos in vitro ${ }^{10-12}$, en animales ${ }^{13,14}$, $\mathrm{y}$ en humanos ${ }^{15-17}$. Dado el preocupante aumento de la prevalencia de consumo de SEAN en los EE.UU., especialmente entre adolescentes y adultos jóvenes, el gobierno de EE.UU. solicitó a la Academia Nacional de Ciencias, Ingeniería y Medicina un estudio sobre las consecuencias del uso de los cigarrillos electrónicos en Salud Pública, informe publicado a comienzos del año $2018^{18}$. En este se concluye que la evidencia sugiere que los cigarrillos electrónicos tienen riesgos para la salud, que serían menores que los cigarrillos convencionales, pero sus efectos a largo plazo, en morbilidad y mortalidad no son claros. También concluyó que el uso de cigarrillos electrónicos en jóvenes aumenta el riesgo que se inicien en consumir cigarrillos convencionales.

\section{Daño pulmonar asociado al uso de cigarrillos electrónicos-vapeadores}

El 30 de agosto de 2019 el Centro para el Control de Enfermedades (CDC) de EE.UU. emitió una alerta ${ }^{19}$ por una importante cantidad de casos de enfermedad respiratoria severa en personas jóvenes, asociada al uso de cigarrillos electrónicos. En septiembre se publicó la primera serie de enfermos de los estados de Illinois y Wis$\operatorname{consin}^{20}$, siguiendo una serie de la Universidad de Pittsburg ${ }^{21}$ y otra del estado de Utah ${ }^{22}$, además de los informes periódicos del CDC a través del 'Reporte Semanal de Morbilidad y Mortalidad' (MMWR). Esta alerta se expandió por todo el mundo y no tardaron en aparecer casos en Canadá, Japón, Brasil, Argentina, Ecuador, Barcelona ${ }^{23}$ y $\mathrm{México}^{24}$.

EL CDC propuso $^{25}$ una definición operacional sobre los casos de enfermedad pulmonar asociada con el uso de cigarrillos electrónicos-vapeadores (EVALI: e-cigarette or vaping associated lung injury), definiendo casos confirmados y casos probables (Tabla 1).

A continuación se presentan las características demográficas, síntomas, evaluación y curso clínico de los primeros 53 pacientes reportados en los estados de Illinois y Wisconsin ${ }^{20}$, siendo estas características muy similares en las publicaciones posteriores $^{21,22}$.

La edad media de los pacientes fue de 19 años, (rango 16 a 53), la mayoría de sexo masculino 44/53 (83\%). El 30\% tenía antecedentes de asma 
Tabla 1. Definición de casos de daño pulmonar asociado al uso de cigarrillos electrónicos-vapeadores: EVALI

\section{Casos confirmados}

- Uso de cigarrillos electrónicos o administración de concentrados de marihuana en los 90 días previos al inicio de los síntomas

- Infiltrados pulmonares tales como opacidades en la Rx de tórax o vidrio esmerilado en TAC de tórax

- Ausencia de infección pulmonar: mínimo panel viral negativo, además de antígeno urinario para Streptococcus pneumoniae y Legionella pneumophila y cultivos negativos (esputo, sangre, LBA)

- No hay evidencias de diagnóstico alternativo plausible (enfermedad cardíaca, reumatológica o neoplásica)

\section{Casos probables}

- Uso de cigarrillos electrónicos o administración de concentrados de marihuana en los 90 días previos al inicio de los síntomas

- Infiltrados pulmonares tales como opacidades en la Rx de tórax o vidrio esmerilado en TAC de tórax

- Infección pulmonar identificada por cultivo o PCR, pero el equipo clínico a cargo del paciente cree que no es la única causa del proceso patológico

- No hay evidencias de diagnóstico alternativo plausible (enfermedad cardíaca, reumatológica o neoplásica)

$\mathrm{LBA}=$ lavado bronquio-alveolar; $\mathrm{Rx}=$ radiografía; $\mathrm{TAC}=$ Tomografía axial computarizada

bronquial y un $34 \%$ de trastornos del ánimo/ ansiedad.

Sobre el uso de cigarrillos electrónicos en los 90 días previos a la enfermedad, lo utilizaron con nicotina el $61 \%$ de los pacientes, sólo con nicotina $17 \%$. Con Tetrahidrocanabinoides (THC) lo usó el 80\% de ellos y sólo con THC el 37\%. Mezclaron nicotina y THC el $44 \%$ de los pacientes.

La duración media de los síntomas antes de consultar fue 6 días. Los síntomas respiratorios se presentaron en el $98 \%$ de los casos, siendo la disnea lo más frecuente ( $87 \%$ ), seguido de tos $83 \%$, dolor torácico $55 \%$, dolor pleurítico $38 \%$ y hemoptisis en $11 \%$. Los síntomas gastrointestinales fueron frecuentes $(81 \%)$, siendo lo más frecuente las náuseas en $70 \%$, vómitos $66 \%$, diarrea $43 \%$ y dolor abdominal $43 \%$. Síntomas generales se presentaron en el $100 \%$ de los pacientes, sensación febril $81 \%$, calofríos $58 \%$ y pérdida de peso en el $26 \%$ de los casos.

El 29\% de los pacientes presentó temperatura $\geq 38^{\circ} \mathrm{C}$, y el $64 \%$ una frecuencia cardiaca $>100$ ciclos/min. Frecuencia respiratoria $>20$ ciclos/ min se encontró en $43 \%$. La saturación de $\mathrm{O}_{2}$ fue normal $(\geq 95 \%)$ en $31 \%$ de los casos. El $38 \%$ tenía entre $89-94 \%$ e hipoxemia franca $(\leq 88 \%)$ en $31 \%$.

En el laboratorio inicial, tenían leucocitosis $>11.000 / \mathrm{mm}^{3}$ el $87 \%$ de los pacientes, neutrófilos $>80 \%$ el $94 \%$ de ellos. Hiponatremia e hipokalemia se constató en $33 \%$ de 53 pacientes.

Hallazgos radiológicos: Rx tórax anormal se constató en $91 \%$ de los casos, TAC de tórax anormal en $100 \%$, con infiltrados bilaterales en Rx o TAC, en el $100 \%$ de los casos.

El $72 \%$ de los pacientes presentaron consultas ambulatorias o al Servicio de Urgencia, resultando hospitalizados el 94\%. La duración de la hospitalización fue de 6 días, fluctuando entre 1 y 25 días. El 87\% de los pacientes recibieron $\mathrm{O}_{2}$ suplementario. El 36\% recibió ventilación mecánica no invasiva (VMNI), requiriendo Intubación y ventilación mecánica invasiva (VMI) el 32\%. Ingresaron a UCI el 58\%, con un desenlace fatal $(2 \%)$.

Tratamiento: En la gran mayoría de los casos (87\%) se utilizaron antibióticos para infección del tracto respiratorio inferior, tanto en forma ambulatoria (45\%), como durante la hospitalización $(90 \%)$. Los corticoides fueron ampliamente utilizados $(92 \%)$ en forma sistémica (vo o iv). Hubo mejoría clínica documentada en el $65 \%$ de los pacientes.

\section{Radiología del daño pulmonar asociado al uso de cigarrillo-e}

Se han encontrado diversos patrones de imágenes radiológicas en estos enfermos. De acuerdo a Henry et al. ${ }^{26}$ considerando 34 casos de EVALI, identificaron al menos 4 patrones asociadas al uso de cigarrillos electrónicos: neumonía eosinofílica aguda, daño alveolar difuso, neumonía organizativa y neumonía lipoidea, además de algunos casos con patrones variegados. Aunque la diversidad de patrones de imágenes sugieren diferentes mecanismos de daño, y probablemente más patrones se continuaran reportando, la mayoría de los patrones presentan consolidaciones predominantemente basales y opacidades en vidrio esmerilado, a menudo con preservación subpleural o lobular. 


\section{Diacetyl (2,3 butanedione) $\mathrm{H}_{3} \mathrm{C}^{\prime}{ }_{0}^{\mathrm{C}}-\mathrm{C}^{\prime}{ }^{\mathrm{C}} \mathrm{H}_{3}$}

Figura 1. Diacetilo o 2,3 butanodiona.

Estos mismos autores amplían la información radiológica en otro artículo ${ }^{27}$ describiendo varias formas de presentación radiológica: neumonitis por hipersensibilidad, hemorragia alveolar difusa, daño alveolar difuso, neumonía eosinofílica aguda, neumonía organizativa, neumonía lipoidea, neumonía intersticial a células gigantes. Agregan que podrían presentarse otras formas dada la heterogeneidad de la estructura de los cigarrillos electrónicos y de las sustancias aerolizadas. Se ha descrito bronquiolitis respiratoria asociada a enfermedad intersticial así como bronquiolitis constrictiva, esta última asociada al uso de los saborizantes que, al calentarse, producen diacetilo (Figura 1), sustancia demostradamente tóxica sobre los pulmones según OMS y OIT ${ }^{40}$.

\section{Patología del daño pulmonar asociado al uso de cigarrillos electrónicos-vapeadores}

Butt et al. ${ }^{28}$ revisaron las biopsias de 17 pacientes. En todos los casos los hallazgos histopatológicos mostraron patrones de daño pulmonar agudo, incluyendo neumonitis fibrinosa aguda, daño alveolar difuso o neumonía organizativa, usualmente bronquiolo céntrica y acompañada de bronquiolitis. Ningún hallazgo histológico fue específico, pero en todos se encontraron macrófagos espumosos y vacuolización de neumocitos. Predominaron los neutrófilos, los eosinófilos eran raros y no se encontraron granulomas. Los hallazgos histológicos no fueron consistentes con neumonía lipoidea exógena, lo cual cuestiona el planteamiento de que el factor causal sería el acetato de Vitamina E, presente en el líquido de los cigarrillos electrónicos ${ }^{29}$. Estos autores plantean que los macrófagos cargados de lípidos con tinción con rojo $\mathrm{O}$ no serían marcadores de toxicidad sino marcadores de exposición.

Posterior a la publicación de Butt, el $\mathrm{CDC}^{30}$ reportó los hallazgos del lavado bronquio-alveolar (LBA) de 29 pacientes de 10 estados de EE.UU. Estudiaron con espectrometría de masas diversos tóxicos específicos que previamente habían sido identificados en los líquidos y dispositivos utilizados para 'vapear' en pacientes con EVALI. Con este procedimiento podían identificar acetato de vitamina $\mathrm{E}$, triglicéridos de cadena media, aceites vegetales (triglicéridos de cadena larga), destilados del petróleo (incluyendo aceite mineral), terpenos, canabinoides y nicotina. Para evaluar la calidad del fluido del LBA midieron dipalmitoilfosfatidilcolina (DPPC), que es el principal fosfolipido del surfactante. La presencia de niveles aceptables de DPPC confirmó que el fluido recuperado del LBA era adecuado en 27 de los 29 pacientes. El acetato de vitamina $\mathrm{E}$ fue encontrado en los 29 casos, en 23 se encontró THC, y en 16 detectaron nicotina. Las otras sustancias estudiadas no se pesquisaron. Los autores concluyen que estos hallazgos proporcionan evidencia directa de la presencia de vitamina E en el sitio del daño de los enfermos con EVALI, pudiendo suponerse una asociación causal, pero es posible que existan más compuestos como causa del daño pulmonar y se necesitan más estudios.

Recientemente se publicaron los resultados de una extensión del estudio de estos mismos autores $^{31}$, con el análisis del LBA en 51 pacientes de EVALI y 99 controles sanos de otro estudio. El acetato de vitamina E fue identificado en 49 de los 51 pacientes (94\%) y no se detectó en los controles sanos. Además detectaron THC y nicotina en el 94 y 64\% de los pacientes, respectivamente.

\section{Características de los pacientes del brote de EVALI en EE. UU.}

Al 17 de enero de 2020 y de acuerdo al último informe del $\mathrm{CDC}^{32}$ publicado a la fecha, se han reportado 2.668 pacientes hospitalizados con EVALI, con 57 fallecidos (2\%). El 66\% eran varones, edad media de 24 años (rango: 13-85) y $76 \%$ eran menores de 35 años. El $82 \%$ reportaron usar productos con THC y $57 \%$ productos con nicotina. $33 \%$ usaron exclusivamente THC y $14 \%$ exclusivamente nicotina. El $41 \%$ uso ambos compuestos. Durante la semana del 15 de septiembre de 2019 ocurrió el 'peak' de hospitalizaciones por EVALI.

E1 95\% de los pacientes con EVALI se hospitalizó. Los pacientes que no se hospitalizaron tenían las mismas características demográficas y usaron los mismos productos que los hospitalizados ${ }^{33}$.

Recientemente se publicaron las características de los pacientes que sufrieron EVALI y que reportaron sólo el uso de productos con nicotina, sin THC. Estos pacientes fueron predominantemente mujeres y de mayor edad. Tuvieron menos 
síntomas constitucionales y leucocitosis que los pacientes que reportaron uso de $\mathrm{THC}^{34}$.

\section{Discusión}

La aparición de este brote de enfermedades respiratorias graves en los Estados Unidos ha producido inquietud en todo el mundo, por la importante cantidad de casos y su gravedad. Contribuye a esta inquietud que hasta el momento no está claro cuál o cuáles sustancias están produciendo este daño pulmonar.

$\mathrm{El}$ acetato de vitamina $\mathrm{E}$ se ha encontrado en todas las muestras de LBA de los pacientes con EVALI, lo que lo hace altamente sospechoso de participar en el daño pulmonar. Pero no hay evidencia suficiente para descartar la contribución de otras sustancias químicas en la génesis de esta patología y probablemente hay más de una causa para este brote de enfermedades respiratorias.

$\mathrm{El}$ acetato de vitamina $\mathrm{E}$ es un producto químico de textura pegajosa y aceitosa que es utilizado como espesante para la elaboración de productos de 'vapeo' que contienen THC. Por otra parte, la vitamina $\mathrm{E}$ se encuentra en muchos alimentos, incluidos aceites vegetales, cereales, carne, frutas y verduras. También está disponible como suplemento alimentario y en muchos productos cosméticos, como cremas para la piel, no habiéndose reportado daños en estas formas de uso.

El acetato de vitamina $E$ es el éster de la vitamina $\mathrm{E}$ (alfa tocoferol) y ácido acético. Diversos estudios $^{31}$ sugieren que puede afectar la capacidad del surfactante para mantener la tensión superficial en los alvéolos pulmonares. Otro efecto posible del acetato de vitamina $E$ es que al calentarse en el líquido del cigarrillo electrónico se produce otro compuesto dañino como el keteno, que tiene el potencial de ser irritante pulmonar, dependiendo de su concentración. Otros autores ${ }^{36}$ postulan que los isómeros de vitamina $\mathrm{E}$ pueden tener efectos regulatorios en la proteína kinasa $\mathrm{C}$ $\alpha(\mathrm{PKC} \alpha)$ en las células endoteliales respiratorias regulando el reclutamiento de leucocitos, una etapa crítica para inducir hiperrespuesta de la vía aérea e inflamación pulmonar.

Independiente de la causa de este brote de enfermedades respiratorias asociadas al cigarrillo electrónico, el Centro de Control y Prevención de Enfermedades (CDC) de los EE. UU. realizó diversas recomendaciones ${ }^{37}$ :

Como la mayoría de los pacientes con EVALI reportaron usar productos que contenían THC, el $\mathrm{CDC}$ recomienda no usar vapeadores con THC, especialmente los procedentes del mercado informal (amigos, familiares, adquiridos por Internet).
También recomienda no modificar ni agregar otros compuestos al cigarrillo electrónico que no estén indicados por el fabricante.

Como la o las causas específicas de EVALI todavía no se conocen, la única forma de no correr riesgos es la abstinencia de todo tipo de vapeadores, mientras continúa la investigación. Independiente de aquello, estos productos no deberían nunca ser usados por adolescentes, adultos jóvenes o mujeres embarazadas. Las personas que no fuman no deberían comenzar a usar cigarrillos electrónicos. Los adultos que están usando vapeadores como ayuda para dejar de fumar no deben volver a fumar cigarrillos convencionales y considerar el uso de los medicamentos aprobados por la FDA (US Food and Drug Administration). Los adultos que continúen usando vapeadores deben ser cuidadosos y monitorearse ellos mismos por síntomas y consultar en su sistema de salud si aparecen síntomas como los descritos en este brote.

\section{¿Qué pasa en Chile?}

De acuerdo a datos del Servicio Nacional de Prevención y Rehabilitación del Consumo de Drogas y Alcohol (SENDA) ${ }^{38}$ el uso de cigarrillos electrónicos en Chile está aumentando de manera significativa, a pesar de estar regulado como producto medicinal. Desde los años 2012 a 2016 su consumo aumentó, expresado como prevalencia vida desde 7,3 a 14,1\%, la prevalecía anual subió de $3,6 \%$ a $6,5 \%$ y la prevalencia mensual de 0,9 a $1,2 \%$. Si bien no son cifras tan altas, lo llamativo es el aumento en su uso, que con toda seguridad se ha incrementado más a la fecha actual.

Al conocerse el brote de enfermedades respiratorias asociadas al consumo de cigarrillos electrónicos en EE. UU., el Ministerio de Salud (MINSAL) instruyó a todas las SEREMIs de salud del país para registrar cualquier caso de enfermedad respiratoria que se pudiera relacionar con el uso de cigarrillos electrónicos. Hasta el momento no se han reportado casos de EVALI.

Al mismo tiempo el MINSAL envió un proyecto de ley al parlamento para regular los cigarrillos electrónicos de forma tal que sean considerados de igual manera que los productos que contiene tabaco. Además recientemente el Ministerio de Hacienda junto al MINSAL, acordaron aplicar a los cigarrillos electrónicos los mismos impuestos con que se gravan los cigarrillos convencionales ('El Mercurio', 19 de diciembre de 2019).

La epidemia de EVALI y de 'vapeo' entre los jóvenes requiere de acciones inmediatas y deci- 
sivas para proteger la salud pública ${ }^{39}$. Por esto deberían redoblarse los esfuerzos por regular y controlar el consumo de ENDS, aplicándoseles todos los controles y normativas de salud y económicas que se aplican a los cigarrillos convencionales, lo que implica principalmente: prohibición de venta a menores, prohibición de publicidad, restricción de uso en lugares cerrados de acceso público, advertencias sobre daños en los envases y que tengan los mismos impuestos que el tabaco convencional.

\section{Bibliografía}

1.- GOTTS J, JORDT S-E, MCCONNELL R, TARRAN $\mathrm{R}$. What are the respiratory effects of e-cigarettes? BMJ 2019; 366: 15275 Disponible en: http://dx.doi. org/10.1136/bmj.15275.

2.- MCNEILL A, BROSE LS, CALDER R, HITCHMAN SC, HAJEK P, MCROBBIE H. E-cigarettes: an evidence update. A report commissioned by Public Health England. Published August 2015 PHE publications gateway number: 2015260 .

3.- WHO Report on the Global Tobacco Epidemic, 2019. Geneva: World Health Organization; 2019. License: CC BY-NC-SA 3.0 IGO. pp c6-59.

4.- BALS R, BOYD J, ESPOSITO S, FORONJY R, HIEMSTRA PS, JIMÉNEZ-RUIZ CA. et al Electronic cigarettes: a task force report from the European Respiratory Society. Eur Respir J 2019; 53: 1801151 [https://doi.org/10.1183/13993003.01151-2018].

5.- BAM TS, BELLEW W, BEREZHNOVA I, JACKSON-MORRIS A, JONES A, LATIF E et al. Summary Position Statement on e-cigarettes(ECs) and electronic nicotine delivery systems(ENDS) 2014 (updated version) The International Union Against Tuberculosis and Lung Disease (The Union).Disponible en: https://www. theunion.org/espanol/lo-que-hacemos/publicaciones/ oficiales. Consultada: 18 de noviembre de 2019.

6.- SCHRAUFNAGEL DE, BLASI F, DRUMMOND MB, LAM DC, LATIF E, ROSEN MJ, et al. Electronic cigarettes. A position statement of the Forum of International Respiratory Societies. Am J Respir Crit Care Med 2014; 190: 611-8.

7.- ZABERT G (ALAT), GAGA M (ERS), JIMÉNEZ RUIZ C (SEPAR), BULJUBACICH D (AAMR), GARCÍA G (AAMR), VÁZQUEZ J (SMNYCT), RAVARA S (ERS), BELLO S (SER CHILE).Cigarrillo electrónico y demás ENDS: posición de sociedades científicas respiratorias. Revista Americana Medicina Respiratoria 2019; 2: 139-45.

8.- BURSTYN I. Peering through the mist: systematic review of what the chemistry of contaminants in electronic cigarettes tells us about health risks. BMC Public Health 14, 18 (2014). https://doi.org/10.1186/14712458-14-18.
9.- LEE MS, ALLEN JG, CHRISTIANI DC. Endotoxin and 1-3- $\beta$-D-Glucan contamination in electronic cigarette products sold in the United States. Environ Health Perspect 2019; 127: 47008. https://doi.org/10.1289/ EHP3469.

10.- GHOSH A, COAKLEY RC, MASCENIK T, ROWELL TR, DAVIS ES, ROGERS K, et al. Chronic E-Cigarette Exposure Alters the Human Bronchial Epithelial Proteome. Am J Respir Crit Care Med 2018; 198: 67-76.

11.- CARSON JL, ZHOU L, BRIGHTON L MILLS KH, ZHOU H, JASPERS I, et al. Temporal structure/ function variation in cultured differentiated human nasal epithelium associated with acute single exposure to tobacco smoke or E-cigarette vapor. Inhal Toxicol 2017; 29: 137-44.

12.- LIN VY, FAIN MD, JACKSON PL, BERRYHILL TF, WILSON LS, MAZUR M, et al. Vaporized ECigarette LiquidsInduce Ion Transport Dysfunction in Airway Epithelia. Am J Respir Cell Mol Biol 2019; 61: 162-73.

13.- LEE HW, PARK SH, WENG MW, WANG HT, HUANG WC, LEPOR H, et al. E-cigarette smoke damages DNA and reduces repair activity in mouse lung, heart, and bladder as well as in human lung and bladder cells. Proc Nat Acad Sci USA 2018; 115: E1560-9.

14.- GARCÍA-ARCOS I, GERAGHTY P, BAUMLIN N, CAMPOS M, DABO AJ, JUNDI B, et al. Chronic electroniccigarette exposure in mice induces features of COPD in a nicotine dependent manner. Thorax 2016; 71: 1119-29.

15.- MCCONNELL R, BARRINGTON-TRIMIS JL, WANG K, URMAN R, HONG H, UNGER J, et al. Electronic Cigarette Use and Respiratory Symptoms in Adolescents. Am J Respir Crit Care Med 2017; 195 : 1043-9.

16.- WILLS TA, PAGANO I, WILLIAMS RJ, TAM EK. E-cigarette use and respiratory disorder in an adult sample. Drug Alcohol Depend 2019; 194: 363-70.

17.- MEO SA, ANSARY MA, BARAYAN FR, ALMUSALLAM AS, ALMEHAID AM, ALARIFI NS, et al. Electronic Cigarettes: Impact on Lung Function and Fractional Exhaled Nitric Oxide Among Healthy Adults. Am J Mens Health 2019; 13: 1557988318806073. doi: $10.1177 / 1557988318806073$.

18.- NATIONAL ACADEMIES OF SCIENCES, ENGINEERING, AND MEDICINE.2018. Public health consequences of e-cigarettes. Washington, DC: The National Academies Press. doi: https://doi. org/10.17226/24952.

19.- CENTERS FOR DISEASE CONTROL AND PREVENTION. Health Alert Network: severe pulmonary disease associated with usinge-cigarette products. August 30, 2019 Disponible en: https://emergency.cdc. gov/ han/ han00421.asp). Consultado: 21 de noviembre de 2019. 
20.- LAYDEN JE, GHINAI I, PRAY I, KIMBALL A, LAYER M, TENFORDE M, et al. Pulmonary Illness Related toE-Cigarette Use in Illinois and Wisconsin Preliminary Report. N Engl J Med 2019. doi:10.1056/ NEJMoa1911614.

21.- TRIANTAFYLLOU GA, TIBERIO PJ, ZOU RH, LAMBERTY PH, LYNCH MJ, KREIT JW, et al. Vaping-Associated Acute Lung Injury: A Case Series. Ama J Respir Crit Care Med 2019; 200: 1430-1.

22.- LEWIS N, MCCAFFREY K; SAGE K, CHIA-JUNG CHENG, GREEN J, GOLDSTEIN L, et al. E-cigarette Use, or Vaping, Practices and Characteristics Among Persons with Associated Lung Injury - Utah, AprilOctober 2019. MMWR Morb Mortal Wkly Rep 2019; 68: 953-6

23.- CASANOVA GS, AMARO R, SOLER N, SÁNCHEZ M, BADÍA J R, BARBERÀ JA, AGUSTÍ A. An imported case of e-cigarette or vaping associated lung injury (EVALI) in Barcelona. Eur Respir J 2019; https:// doi.org/10.1183/13993003.02076-2019.

24.- MACIAS A, GARCÍA F, SALDANA S. A patient from Mexico with vaping-associated lung injury, seizures and renal failure. Tob Induc Dis 2019; 17: 91 doi: $10.18332 /$ tid/114316.

25.- SCHIER JG, MEIMAN JG, LAYDEN J, MIKOSZ CA, VANFRANK B, KING B A, et al. Severe Pulmonary Disease Associated with Electronic-Cigarette-Product Use-Interim Guidance. MMWR Morb Mortal Wkly Rep 2019; 68: 787-90. DOI: http://dx.doi.org/10.15585/ mmwr.mm6836e2external icon.

26.- HENRY TS, KANNE JP, KLIGERMAN SJ. Imaging of vaping associated lung injury. N Engl J Med 2019; 381 (15): 1486-7.

27.- HENRY TS, KLIGERMAN SJ, RAPTIS CA, MANN $\mathrm{H}$, SECHRIST JW, KANNE J. Imaging finding of vaping-associated lung injury. Am J Radiol 2020; 214: $1-8$.

28.- BUTT YM, SMITH ML, CECCHINI MJ, BOLAND JM, KHOOR A, LARSEN BT, et al. Pathology of vaping associated lung injury. N Engl J Med 2019. DOI: 10.1056/NEJMc1913069.

29.- MADDOCK SD, CIRULIS MM, CALLAHAN SJ, KEENAN LM, PIROZZI CS, RAMAN SM, et al. Pulmonary lipid laden macrophages and vaping. N Engl J Med 2019; 381: 1488-9. DOI: 10.1056/NEJMc1912038.

30.- BLOUNT BC, KARWOWSKI MP, ESPINOSA MM, REES J, SOSNOFF C, COWAN E et al. Evaluation of Bronchoalveolar Lavage Fluid from Patients in an Outbreak of E-cigarette, or Vaping, Product Use-Associated Lung Injury - 10 States, August-October 2019. MMWR Morb Mortal Wkly Rep 2019; 68: 1040-1.

31.- BLOUNT BC, KARWOWSKI MP, SHIELDS PG, MOREL-ESPINOSA M, VALENTIN-BLASINI L, GARDNER M. et al; for the Lung Injury Response Laboratory Working Group. Vitamin E Acetate in
Bronchoalveolar-Lavage Fluid Associated with EVALI. N Engl J Med 2020; 382 (8): 697-705. DOI: 10.1056/ NEJMoa1916433.

32.- KRISHNASAMY VP, HALLOWELL BD, KO JY, BOARD A, HARTNETT K, SALVATORE PP, et al. Update: Characteristics of a Nationwide Outbreak of E-cigarette, or Vaping, Product Use-Associated Lung Injury-United States, August 2019-January 2020. MMWR Morb Mortal Wkly Rep 2020; 69: 90-4.

33.- CHATHAM-STEPHENS K, ROGUSKI K, JANG Y, CHO P, JATLAOUI TC, KABBANI S, et al. Characteristics of Hospitalized and Nonhospitalized Patients in a Nationwide Outbreak of E-cigarette, or Vaping, Product Use-Associated Lung Injury-United States, November 2019. MMWR Morb Mortal Wkly Rep 2019; 68: 1076-80.

34.- GHINAI I, NAVON L, GUNN JK, DUCA LM, BRISTER S, LOVE S, et al. Characteristics of Persons Who Report Using Only Nicotine-Containing Products Among Interviewed Patients with E-cigarette, or Vaping, Product Use -Associated Lung Injury- Illinois, August-December 2019. MMWR Morb Mortal Wkly Rep 2020; 69 (3): 84-9. doi: 10.15585/mmwr. mm6903e1.

35.- MIKOSZ CA, DANIELSON M, ANDERSON KN, POLLACK LA, CURRIE DW, NJAI R, et al. Characteristics of Patients Experiencing Rehospitalization or Death After Hospital Discharge in a Nationwide Outbreak of E-cigarette, or Vaping, Product Use -Associated Lung Injury- United States, 2019. MMWR Morb Mortal Wkly Rep 2020; 68: 1183-8.

36.- LAL A, MISHRA AK, SAHN KK. Vitamin E acetate and e-cigarette or vaping product-associated lung injury (EVALI): an update. Disponible en: https://doi. org/10.1016/j.amjmed.2019.11.005

37.- JATLAOUI TC, WILTZ JL, KABBANI S, SIEGEL DA, KOPPAKA R, MONTANDON M, et al. Update: Interim Guidance for Health Care Providers for Managing Patients with Suspected E-cigarette, or Vaping, Product Use -Associated Lung Injury- United States, November 2019. MMWR Morb Mortal Wkly Rep 2019; 68: 1081-6.

38.- SENDA. Décimo Segundo Estudio Nacional de Drogas en Población General, 2016. ISBN: 978-956-9141-54-6.

39.- KING BA, JONES CM, BALDWIN GT, BRISS PA. The EVALI and Youth Vaping Epidemics-Implications for Public Health. N Engl J Med 2020; 382 (8): 689-91.

40.- ILO. 2,3 Butanodiona. Consultado: 23 marzo 2020. Disponible en: http://www.ilo.org/dyn/icsc/showcard. display?p_card_id=1168\&p_version=1\&p_lang=es.

Correspondencia a:

Dr. Sergio Bello S.

Comisión de Tabaquismo

Sociedad Chilena de Enfermedades Respiratorias

Email: sbello.sbs@gmail.com 